\title{
3, 6 or 9-band shooters: tailor made ligators as needed
}

\author{
Sandeep Nijhawan · Gaurav Gupta $\cdot$ Anil Sharma • \\ Amit Mathur • Bharat Sapra $\cdot$ Subhash Nepalia
}

Published online: 27 May 2011

(C) Indian Society of Gastroenterology 2011

Patients with portal hypertension require esophageal variceal ligation for primary or secondary prophylaxis of variceal bleed. These patients have grade II to IV varices, the number of columns are variable from 1 to 4 . The length of the columns may be short, or may extend into the upper part of esophagus.

The number of bands required varies according to the grade, number and length of esophageal varices. Most of the commercially available ligators have seven bands for ligation (Speedband, Boston Scientific, USA; Vu-max multiband ligator, Advanced Medicure System, India). This leads to wastage of bands if the number of bands required is less than six. Also, patients requiring more than six bands would require more than one ligator set and repeated insertion of the scope. Since all patients are subjected to a diagnostic endoscopy, one can often predict the number of bands required for ligation of all varices.

We have indigenized a single thread system for preparation of ligators [1,2]. These bands are effective and provide better vision during procedure as compared to double-thread systems available commercially [2]. This requires less thread to be accommodated over the cylinder in between the bands and leaves more space for greater number of bands to be put over the cylinder. Therefore, we indigenized a ligator which can shoot nine bands with the same six shooter cylinder thereby giving us different ligators shooting three, six or nine bands with a single thread system (Fig. 1).

We choose the ligator according to the predicted bands required while doing diagnostic endoscopy. Three-shooter ligator is used for patients with smaller and/or lesser number of varices, which frequently happens after the first or second session of ligation.

S. Nijhawan $(\bowtie) \cdot$ G. Gupta $\cdot$ A. Sharma $\cdot$ A. Mathur $\cdot$ B. Sapra

S. Nepalia

Department of Gastroenterology, S M S Medical College,

112, Panchsheel Enclave, Gokul Bhai Bhatt Marg, Durgapura, Jaipur, India

e-mail: dr_nijhawan@yahoo.com

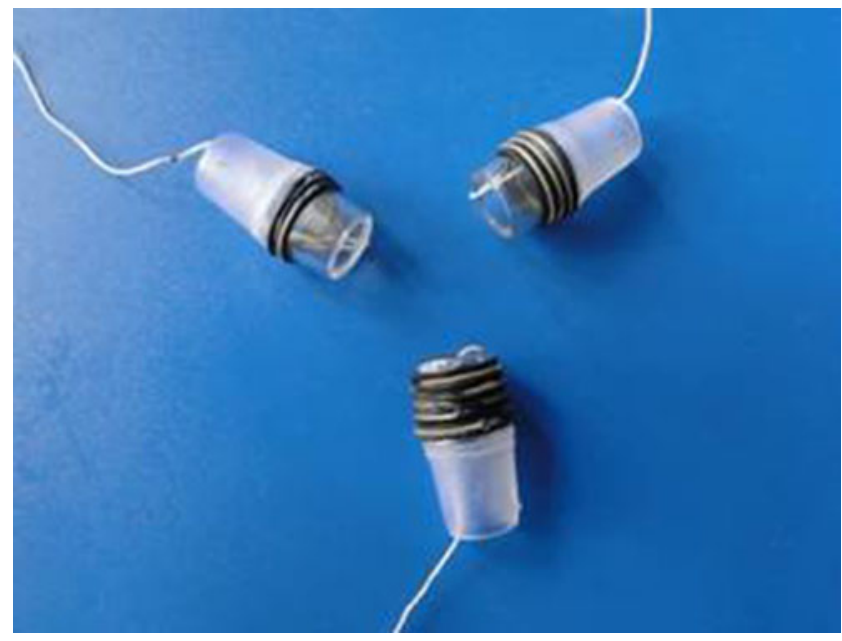

Fig. 13,6 and 9 band shooters

Patients with larger and longer varices at first session would require a nine-shooter ligator, this helps to avoid multiple introductions and discomfort.

The cost of the ligators ranges from Rs 700 (US\$ 14 [1 US\$=Rs 50]) for the 3-band shooter to Rs 900 (US\$ 18) for the 9-band shooter.

Thus, we find that using ligators with different number of bands decreases wastage of bands in patients of esophageal varices requiring ligation.

\section{References}

1. Nijhawan S, Joshi A, Shende A, Mathur A, Rai RR. A new band deployment system: do we really need two threads? Endoscopy. 2005;37:495.

2. Nijhawan S, Jain P, Singla D, Mallikarjun P, Singh V. Single-thread multishooter ligator system shooting at the 7 o'clock position: an ideal band ligator. Endoscopy. 2007;39 Suppl 1:E166. 